\title{
Justiça Organizacional, Estresse e o Conflito Trabalho - Família: Os Efeitos da Tríade em uma Prefeitura do Sul do Brasil
}

\section{Organizational Justice, Stress and the Workplace - Family Conflict: The Effects of the Triad in a City Hall in Southern Brazil}

\author{
Ronaldo Leão de Miranda \\ https://orcid.org/0000-0001-6778-2463 \\ Péricles Ewaldo Jader Pereira \\ https://orcid.org/0000-0002-4407-7398 \\ Antonio Ronaldo Madeira de Carvalho \\ https://orcid.org/0000-0002-9558-1068 \\ Luciano Castro de Carvalho \\ https://orcid.org/0000-0002-1740-6053
}

\author{
Mestre em Desenvolvimento Regional. Universidade Regional de Blumenau (FURB) - Brasil. \\ ronaldo_leaomiranda@hotmail.com \\ Mestre em Administração. Universidade Regional de Blumenau (FURB) - Brasil. \\ peckjader@gmail.com \\ Mestre em Administração. Universidade Regional de Blumenau (FURB) - Brasil. \\ ronaldo.madeira00@hotmail.com \\ Doutor em Administração. Universidade Regional de Blumenau (FURB) - Brasil. \\ luccar@gmail.com
}

\section{RESUMO}

A percepção de justiça organizacional tem se mostrado uma variável de grande poder heurístico para explicar comportamentos, atitudes e eficácia nas organizações. O local de trabalho tem se tornado cada vez mais um campo de estresse, injustiças e conflitos, sendo assim, buscou-se analisar a influência da justiça organizacional sobre o estresse e sobre o conflito trabalho - família, tendo como objeto de estudo, uma prefeitura da região sul do Brasil. Metodologicamente, a pesquisa caracteriza-se como exploratória em relação a seu objetivo, quanto aos procedimentos pode ser considerada como pesquisa aplicada a partir do levantamento de dados primários e quantitativa em relação à abordagem do problema, sendo analisada por meio de técnicas estatísticas (MEE). Quanto aos dados, percebeu-se que os servidores da prefeitura estudada, sentem-se mais estressados quando as ações ou os mecanismos referentes à remuneração, ao processo de tomada de decisão e questões interpessoais são injustas. Em um segundo momento, quando os servidores percebem que as ações ou os mecanismos são injustos dentro do ambiente organizacional, isso gera o estresse, que por sua vez gera o conflito em casa pelas questões do trabalho. Por fim, neste estudo não se evidenciou por meio da modelagem de equações estruturais (MEE) que a justiça organizacional tem influência significativa nas relações de conflito trabalho - família, dadas atuais percepções sobre as condições de trabalho dentro da prefeitura estudada.

Palavras-Chave: Justiça Organizacional, Estresse, Conflito Trabalho - Família, Prefeitura, Brasil.

\begin{abstract}
The perception of organizational justice has proven to be a variable of great heuristic power to explain behaviors, attitudes and effectiveness in organizations. The workplace has increasingly become a field of stress, injustice and conflict, therefore, we sought to analyze the influence of organizational justice on stress and on the conflict between work-family, having as object of study, a city hall of South region of Brazil. Methodologically, the research is characterized as exploratory in relation to its objective, as for the procedures it can be considered as applied research from the survey of primary data and quantitative in relation to the approach of the problem, being analyzed by means of statistical techniques (Structural equation Modeling). As for the data, it was noticed that the employees of the studied city hall, feel more stressed when the actions or mechanisms related to remuneration, decision-making process and interpersonal issues are unfair. In a second moment, when the servers realize that the actions or mechanisms are unfair within the organizational environment, this creates stress, which in turn generates conflict at home due to work issues. Finally, in this study, it was not evident through structural equation modeling (SEM) that organizational justice has a significant influence on conflict between work-family, given current perceptions about working conditions within the studied city.
\end{abstract}

Keywords: Organizational Justice, Stress, Work - Family Conflict, City Hall, Brazil. 


\section{INTRODUÇÃO}

Desde 1960, com o surgimento dentro do campo da psicologia, que a psicologia social da justiça vem se preocupando em demonstrar o papel crucial que os valores, as crenças e os sentimentos têm em sobre o que é justo. Assim, a percepção de justiça organizacional tem se mostrado uma variável de grande poder heurístico para explicar comportamentos, atitudes e eficácia nas organizações (Prestes, Mendonça \& Ferreira, 2013). Atualmente, questões de ordem subjetiva, como as que estão envolvidas nos julgamentos de justiça, têm representado foco de interesse primordial no campo de estudos das relações entre funcionários e seus empregadores. No campo das ciências sociais aplicadas, mais precisamente na administração, estudos já evidenciaram que a justiça organizacional pode afetar as atitudes e comportamentos dos funcionários (Judge \& Colquitt, 2004). Para Monteiro \& Mourão (2016) a percepção de justiça não se refere apenas à distribuição justa dos recursos, mas a participação dos trabalhadores nos processos de tomada de decisão.

Diante das inúmeras definições conceituais de justiça organizacional, destacam-se alguns autores clássicos (Adams, 1963; Greenberg, 1993; Hoffe, 2003, Rawls, 2008), bem como das subcategorias de justiça (Distributiva, Procedimental e Interacional) (Adams, 1965; Lind \& Tyler, 1988; Rego, 2000). Recentemente, alguns estudos de Dal Corso, De Carlo, Carluccio, Girardi \& Falco (2019), Gu, Nolan \& Rowley (2019) e Correia, Mendes \& Silva (2019), analisaram que as percepções de justiça, indicam que os funcionários que são tratados de maneira justa por seus superiores, tendem a ser mais propensos a aceitar as decisões, além de apresentar um comprometimento socialmente favorável, com maior compromisso e bem-estar no trabalho (Lim \& Loosemore, 2017).

Em outros casos, constatou-se que a percepção de injustiça organizacional é um dos fatores que contribuem para o estresse no trabalho em decorrência dos níveis de tensão, que por sua vez podem causar reações físicas e psicológicas (Elovainio, Kivimaki \& Vahtera, 2002; Omar, 2006). Quando se trata do tema estresse, alguns autores clássicos são bem vindos para a discussão (Selye, 1965; Karasek \& Theorell, 1990; Paschoal \& Tamayo, 2004). Como é conhecido pela literatura, cada indivíduo possui uma forma de lidar com o estresse. Estresse esse, que são os principais determinantes para o conflito trabalho - família, que por sua vez também é um dos fatores causadores do estresse (O'Driscoll et al, 2006; Molino et al, 2015).

A percepção de um ambiente organizacional justo promove atitudes positivas, bem como, ambientes injustos promovem atitudes negativas (Prestes, Mendonça \& Ferreira, 2013). No que tange a justiça organizacional no setor público, destaca-se o estudo de Da Rocha, Ceretta \& Andretto (2016), o qual foi verificado a percepção de justiça dos servidores de uma instituição pública da esfera judiciária estadual localizada no Estado do Paraná, e o principal resultado indica que (44\%) dos servidores indicaram estar insatisfeitos com os procedimentos utilizados pela instituição. Portanto, percebe-se que os estudos empíricos realizados tanto no setor privado quanto público, possibilitaram que a justiça organizacional se desenvolvesse como um conceito multidimensional, em que novas dimensões e relações surgem com o desenvolvimento e aprofundamento de novos estudos (Ahmadi, Daraei, Rabiel, Salamzadeh \& Takallo, 2012; Beuren, Dos Santos, Marques \& Resendes, 2017).

A partir dos estudos citados, se ressalta a importância de estudar as instituições públicas, ainda há muito a se acrescentar ao campo da justiça organizacional, principalmente quando se coloca em discussão o estresse e o conflito trabalho família. Partindo destas breves reflexões, a pergunta norteadora a ser respondida neste estudo, diz respeito a verificar: Em que medida a justiça organizacional influencia o estresse e o conflito trabalho - família, tendo como objeto de estudo uma prefeitura localizada na região sul do Brasil? Logo, aponta-se para o objetivo principal, que é analisar a influência da justiça organizacional sobre o estresse e sobre o conflito trabalho - família, tendo como objeto de estudo, uma prefeitura da região sul do Brasil.

A contribuição deste estudo consiste em reforçar as discussões que estão sendo feitas no campo da justiça organizacional, incrementando a discussão ao colocar o estresse e o conflito trabalho - família em análise. Com isso, é possível identificar a percepção de justiça dos servidores públicos, bem como identificar se o ambiente de trabalho público gera estresse e consequentemente conflito trabalho - família, tendo em vista que o serviço público possui em muitos casos vieses políticos na tomada de decisão. Destarte, o trabalho está dividido em cinco seções, sendo composto primeiramente por esta breve introdução; seguido pelo referencial teórico, pelos procedimentos metodológicos; apresentação e análise dos resultados e, por fim, as considerações finais.

\section{REFERENCIAL TEÓRICO}

Um dos caminhos que formatou o termo justiça organizacional (JO), originou-se através das pesquisas realizadas por Homans (1961). A investigação de Homans vinculou-se à psicologia social, quando postulou a 
necessidade de proporcionalidade entre recompensas e investimentos em uma relação de troca entre indivíduos (Homans, 1961). E após esse período a JO tem sido investigada em outras áreas como a psicologia organizacional, gestão de recursos humanos e no comportamento organizacional (Lim \& Loosemore, 2017; Garcia; Macadar \& Luciano, 2018; Carluccio, Girardi \& Falco, 2019). Uma segunda corrente que trata o termo justiça organizacional, começou a tomar forma na década de 1965 com John Stacy Adams ao tratar a Teoria da Equidade. A teoria traz em seu cerne a percepção de indivíduos quanto a seu tratamento em ações e comportamentos desempenhados com similaridade. Esta percepção desperta um juízo, onde o indivíduo estabelece comparações de igualdade ou não, com outros sujeitos que desempenham ações semelhantes (Adams, 1965).

A justiça organizacional é proposta como diretrizes orientativas que especificam os direitos e os deveres nas organizações. Esses aspectos são definidos por meio de normas, regras e procedimentos, com o objetivo de apresentar os critérios de distribuição de recursos, responsabilidades, recompensas e encargos aos indivíduos (Garcia; Macadar \& Luciano, 2018). Sabe-se que as organizações são constituídas por pessoas, que segundo Assmar, Ferreira \& Souto (2005), possuem percepções distintas quanto as formas de tratamento entre indivíduos. Em detrimento disso, sua percepção de justiça também é observada de maneira diferente. Essas percepções, sentimentos e pensamentos, para Colquitt (2001), afetam os indivíduos, pois neles criam-se julgamentos quanto a justiça ou injustiça aferida no ambiente, interferindo na convivência das pessoas, em seus grupos sociais e profissionais.

Neste contexto, Colquitt (2001), salienta que a justiça é fundamental para a compreensão do comportamento humano dentro das organizações e que isso está ligado aos resultados organizacionais como satisfação no trabalho, comprometimento organizacional, comportamento de cidadania organizacional e desempenho. Em um contraponto, na esfera empresarial, os funcionários que percebem o tratamento justo por seus superiores (Tepper, 2001; Lim \& Loosemore, 2017), são mais propensos a aceitar as decisões deles advindas, apresentam comprometimento socialmente favorável, além de maior compromisso com suas reponsabilidades profissionais. Diante dessas diferenças de sentimentos e pensamentos, Greenberg (1986), analisou a teoria da justiça segundo as percepções distributivas e processuais/procedimentais.

A teoria da justiça segundo as percepções interpessoais e informacionais, foram analisadas por Greenberg (1993). As tipologias de justiça são discutidas na literatura tendo um conceito amplamente aceito e dividido em quatro vertentes (Beuren, Dos Santos, Marques \& Resendes, 2017), justiça distributiva, processual / procedimental, interpessoal e informacional (Lim \& Loosemore, 2017). Segundo, Greenberg (1986), a justiça distributiva diz respeito as avaliações recebidas em relação ao trabalho realizado, ou seja, aquela que lida com a justiça como resultado (Hartman; Yrle \& Galle, 1999).

De acordo com Colquitt et al (2001), esse contexto é estabelecido, pois a percepção de justiça está conectada com os resultados organizacionais, como satisfação no trabalho, comprometimento organizacional, comportamento organizacional e desempenho (Garcia; Macadar \& Luciano, 2018). Na perspectiva processual, Cropanzano, Bowen \& Gilliland (2007), complementam que os colaboradores ao perceberem que os processos organizacionais são justos, suas ações tenderão a demonstrar mais lealdade e disposição para agir de acordo com os interesses da organização (Garcia; Macadar \& Luciano, 2018). Assim, entende-se que procedimentos claros e justos geram confiança e comprometimento por parte do indivíduo, e que por sua vez, afetam positivamente a execução da estratégia, do desempenho e dos resultados organizacionais. Na visão da justiça interpessoal, as ações demonstram a qualidade da interação, se referindo ao tratamento com que os gestores se dirigem aos funcionários (forma respeitosa e digna) (Al-Zu'Bi, 2010).

Ferreira et al (2018), destacam que existe uma forte propensão à exaustão emocional quando os indivíduos possuem afetos negativos dirigidos ao trabalho, tais como, sentimento de tristeza, angústia e descontentamento, fazendo com que os indivíduos estejam mais vulneráveis a sentir desgaste físico e emocional. No estudo de Maffia \& Pereira (2014) se analisou o nível de intensidade do estresse ocupacional em 617 gestores públicos, e identificou-se que 74\% apresentavam quadro de estresse, variando de leve a moderado $(43,0 \%)$, estresse intenso $(26,0 \%)$ e muito intenso $(5,0 \%)$. Somente $26 \%$ dos gestores não apresentaram manifestações de estresse.

Diante das abordagens conceituais, formula-se a primeira hipótese da pesquisa, considerando a justiça organizacional uma possível relação negativa com o estresse. Assim tem-se a hipótese 1:

\section{$\checkmark \quad$ H1: A percepção de justiça organizacional afeta negativamente o estresse.}

O estresse é um mecanismo usado pelos indivíduos como resposta a circunstâncias que causaram significativo grau de exigência, desequilíbrio, desconforto e injustiça na organização (Ferreira et. al, 2018). Porém, estudos sobre estresse, tem ganhado força e relevância devido à aceleração das mudanças organizacionais e às tendências contemporâneas de reestruturação do trabalho e do emprego e da 
implementação de modelos de gestão (Bohle, Quinlan \& Mayhew, 2001). Atrelada a essas mudanças, se insere a deterioração da qualidade de vida dos indivíduos, repercutindo em uma rotina diária, cada vez mais desgastante e no aumento das situações consideradas estressoras (Chanlat, 2005; Rossi, Perrewé \& Sauter, 2005; Zille, 2011). Assim sendo, o estresse ocupacional está relacionado ao processo de como o indivíduo percebe demandas de trabalho como estressores, os quais, ao extrapolar sua habilidade de enfrentamento, provocam no individuo reações negativas (Paschoal \& Tamayo, 2004).

Cooper, Sloan \& William (1988), apresentam um modelo o qual o estresse é analisado a partir da relação entre a organização e indivíduo, considerando os fatores imbricados em cada um deles. Neste caso, o sujeito percebe as fontes causadoras do estresse e, de acordo com a sua propensão a esse estresse, definida por características de sua personalidade, tende a apresentar sintomas que o levará a doenças físicas e mentais, bem como a disfunções organizacionais (Cooper; Sloan \& William, 1988). Na (figura 1) é possível observar o que foi descrito acima, com o modelo de estresse desenvolvido no final dos anos de 1980, por Cooper et al (1988).

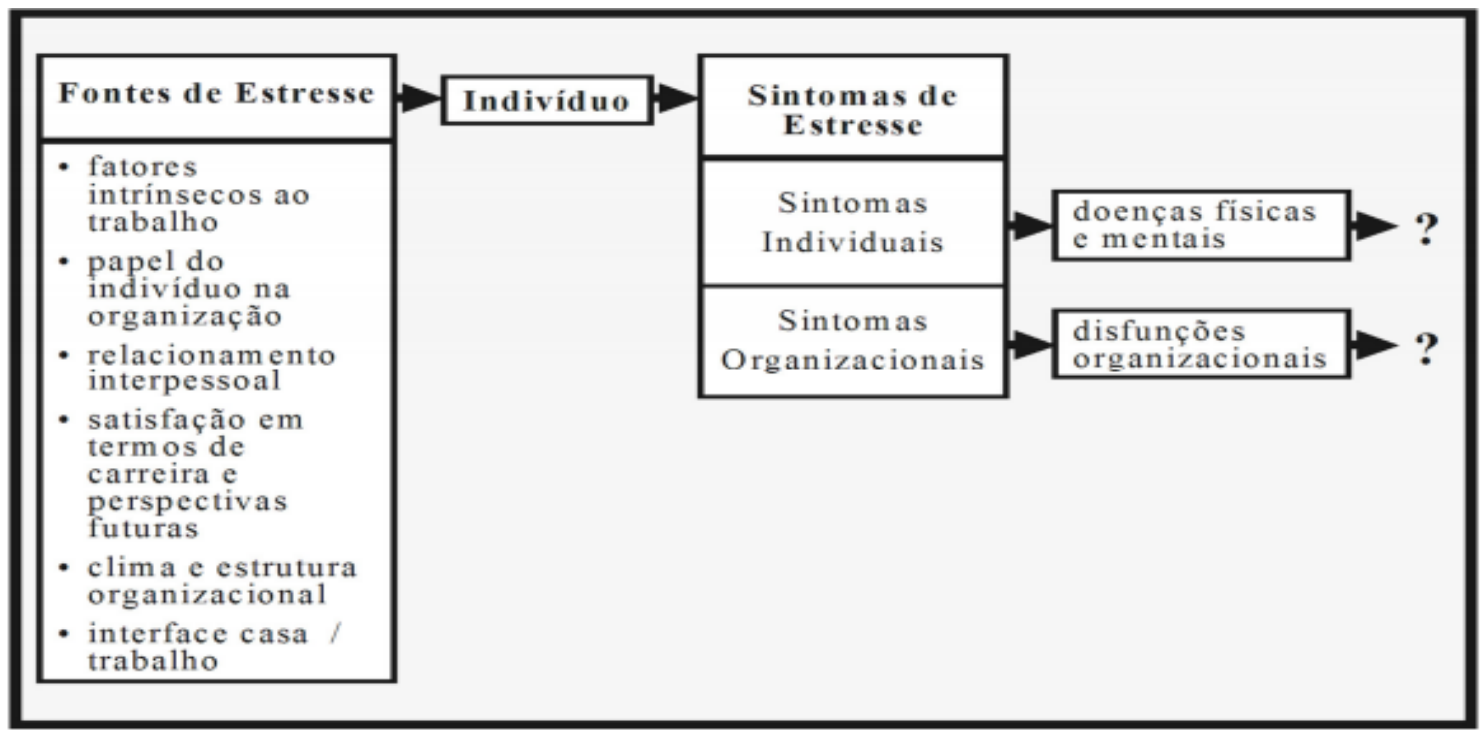

Figura 1. Modelo de estresse de Cooper, Sloan e William (1988)

Fonte: Adaptação de Ferreira (2018) do modelo de Cooper; Sloan; William (1988).

Para Cooper et al (1988), existem seis fontes de estresse (fatores intrínsecos ao trabalho; papel do indivíduo na organização; relacionamento interpessoal; satisfação em termos de carreira e perspectivas futuras; clima e estrutura organizacional; e interface casa - trabalho). Assim, tais fontes estabelecem uma relação com os aspectos pessoais do indivíduo que por sua vez geram sintomas individuais e organizacionais, causando assim doenças físicas, mentais bem como disfunções organizacionais (Cooper; Sloan \& William, 1988). Portanto, o estresse pode estar associado aos fatores do trabalho que excedem a capacidade dos indivíduos às respostas fisiológicas, psicológicas e comportamentais das pessoas aos estressores.

Sousa, Paúl \& Queirós (2020), descobriram em seu estudo com profissionais da área da saúde que existem correlações positivas entre fadiga, stress, burnout e conflito trabalho - família, o que reforça a importância de uma adequada conciliação entre o trabalho e a família e recomendam que as organizações de modo geral (públicas ou privadas) devem contribuir e assumir um papel essencial na identificação e prevenção destes fenómenos.

Diante deste fato, a grande preocupação é identificar os estressores, que são aqueles eventos que provocam respostas indicativas de estresse que interferem no bem-estar, no desempenho e até mesmo como gerador da relação conflito trabalho - família. Algumas das principais fontes de estresse são (i) o excesso de atividades, (ii) a falta de planejamento e, (iii) e os conflitos de interesses e valores (Sadir \& Lipp, 2009). Assim, com vistas a fazer um link com a justiça organizacional e o conflito trabalho - família, o estresse surge na hipótese 2 com o papel de mediador:

\section{$\checkmark \quad$ H2: A percepção de justiça organizacional mediada pelo estresse afeta positivamente $o$} conflito trabalho - família. 
O modelo conceitual do conflito trabalho - família tem origem na teoria dos papéis (Merton, 1957). 0 tema conflito trabalho - família foi definido como uma forma de conflito entre papéis, em que são afetados os resultados dos domínios profissional e familiar, por serem mutuamente incompatíveis em alguns aspectos. Isto é, participação do papel do trabalho (família) é dificultado em virtude da participação no papel da família (trabalho) (Greenhaus \& Beutell, 1985). O conflito trabalho - família pode ser definido como o processo no qual o funcionamento de um indivíduo no domínio do trabalho (ou da família) é influenciado por pressões e recursos do domínio da família (ou do trabalho) (Bakker et al, 2011).

Neste caso, o trabalho influencia na família e/ou a família influencia no trabalho. Para Edwards \& Rothbard (2000), um dos consensos da sociedade civil atual é de que o trabalho diz respeito as atividades instrumentais que têm no seu centro a produção, tanto no sentido objetivo (bens manufaturados, serviços) quanto no sentido subjetivo (significado, afeto), além da primazia de gerar recursos ou condições para suporte da vida individual e familiar.

O entendimento de família, por sua vez, também não é definido de forma consensual. Esse é um conceito complexo, uma vez que abarca laços biológicos, afetivos e costumes sociais que envolvem as pessoas, além de representar um papel social, o qual demarca fronteira entre o mundo doméstico e o mundo externo, bem como a divisão entre o público e o pessoal (Biroli, 2014). Entre os modelos teóricos, que abordam a interface trabalho - família, dois encontram destaque, o primeiro é a perspectiva do conflito dominado durante a maior parte dos anos 80 e 90, e o segundo, o conflito trabalho - família com foco na necessidade de enriquecimento, se tornando cada vez mais em destaque nas últimas duas décadas (Ghislieri et al, 2011; Russo \& Buonocore, 2012).

Nesse sentido, a cultura organizacional, o estilo de liderança e as atitudes da gerência também seriam fatores importantes na busca pelo equilíbrio entre as demandas do trabalho e da família. A percepção da existência desse suporte organizacional agiria como um potencializador da noção de direitos do trabalhador, reduzindo o sacrifício da vida familiar e o estresse, o que poderia produzir maior satisfação no trabalho (Monteiro \& Daniel, 2017).

Tendo em vista a complexidade que é ter um bom desempenho tanto no papel trabalho quanto no papel família, surge a terceira hipótese da pesquisa a ser testada, buscando relacionar justiça organizacional com o conflito trabalho - família. Neste caso, com vista que a justiça organizacional possui um papel de destaque no campo organizacional, essa hipótese infere-se uma relação negativa com o conflito trabalho família.

$\checkmark$ H3: A percepção de justiça organizacional afeta negativamente o conflito trabalho -família.

\section{PROCEDIMENTOS METODOLÓGICOS}

Metodologicamente, a pesquisa caracteriza-se como exploratória em relação a seu objetivo, quanto aos procedimentos pode ser considerada como pesquisa aplicada a partir do levantamento de dados primários e quantitativa em relação à abordagem do problema, sendo analisada por meio de técnicas estatísticas, modelagem de equações estruturais (MEE). Quanto aos respondentes da pesquisa, para se chegar na amostra, seguiu o destacado por Oliveira (2003), que com um nível de confiança de $95 \%$ chegou-se a 218 servidores efetivos de uma população de 1000 servidores públicos. Com esse número de respondentes e quantidade de observações por pergunta (Hair et al, 2010), foi possível conhecer os efeitos da tríade no objeto de estudo (prefeitura)

A coleta de dados foi realizada no período de junho a setembro de 2019, através de um questionário on-line, auxiliado pela ferramenta Google Docs, o qual foi constituído por um conjunto ordenado de perguntas sobre as três dimensões (justiça organizacional, estresse e conflito trabalho - família). O questionário aplicado, foi construído com base nos estudos de Judge \& Colquitt (2004), que avaliou esta mesma tríade, em universidades; o de Rego \& Souto (2004), que olhou a percepção de justiça como antecedente do comprometimento organizacional; e o de Paschoal \& Tamayo (2004), que validou a escala de estresse no trabalho (quadro 1).

Considerando as variáveis apresentadas acima, dispõe-se do seguinte modelo estrutural de pesquisa. 


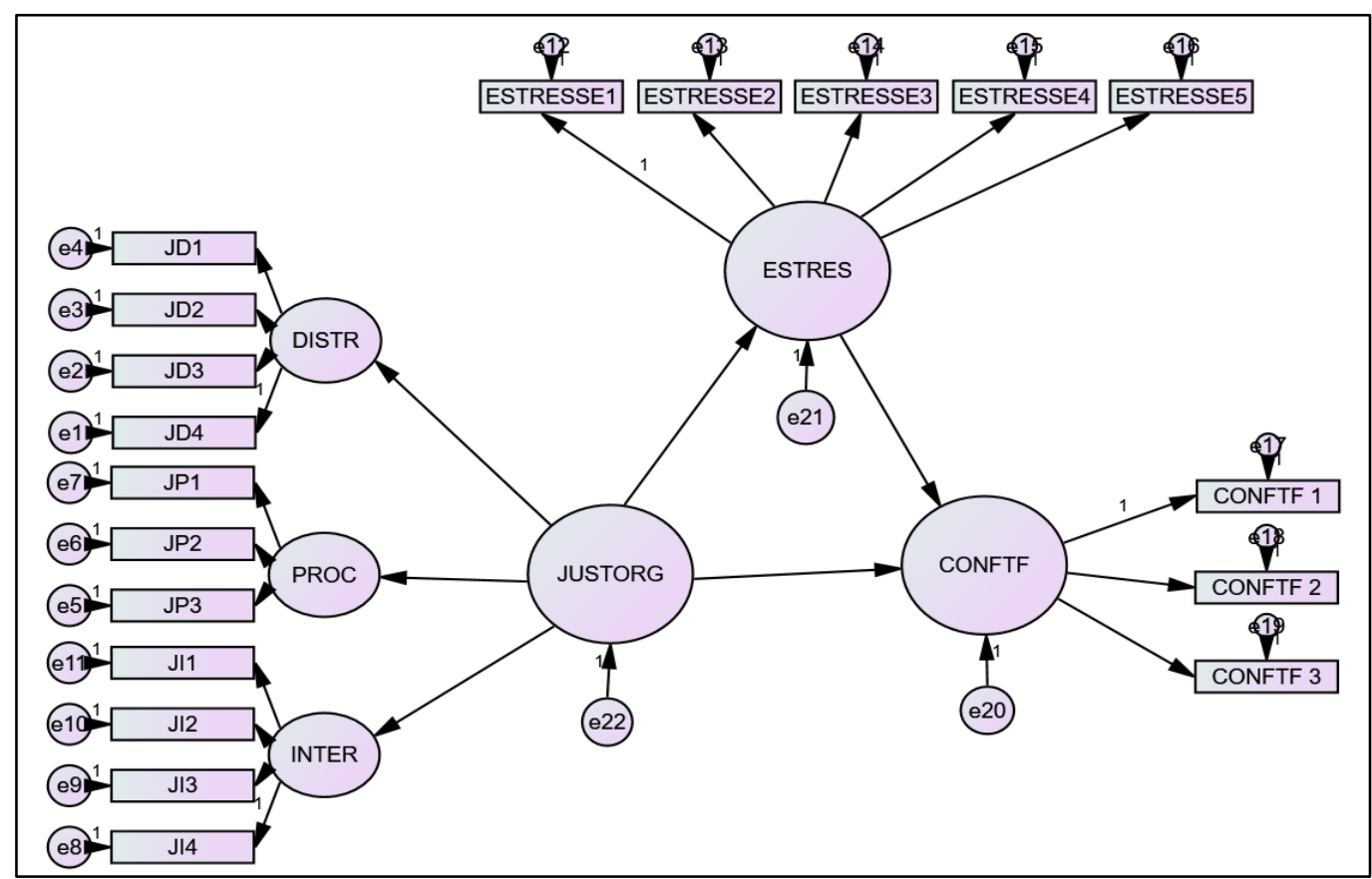

Figura 2. Modelo Estrutural da Pesquisa

Fonte: Dados da pesquisa (2019).

No modelo estrutural de pesquisa (figura 2), se demonstra de forma gráfica as três subdimensões que formam a justiça organizacional com suas devidas variáveis, o qual foram correlacionadas com as cinco variáveis da dimensão estresse e com as três variáveis da dimensão conflito trabalho - família. Logo foi utilizado a dimensão estresse como mediadora, em uma relação indireta, verificando a influência da justiça organizacional sobre o conflito trabalho - família. O quadro 1 apresenta as dimensões da pesquisa, respectivos autores e escala.

\begin{tabular}{|c|c|c|}
\hline DIMENSÕES & ADAPTADO & ESCALA DE RESPOSTAS \\
\hline $\begin{array}{c}\text { Justiça Organizacional - JUSTORG } \\
\text { (11 perguntas) }\end{array}$ & Judge \& Colquitt (2004) & \multirow{3}{*}{$\begin{array}{c}1 \text { = discordo totalmente; } \\
2=\text { discordo moderadamente; } 3 \\
\text { discordo levemente; } \\
4=\text { nem concordo, nem discordo; } \\
5 \text { = concordo levemente; } \\
6=\text { concordo moderadamente; } \\
7 \text { = concordo totalmente }\end{array}$} \\
\hline $\begin{array}{l}\text { Conflito Trabalho - Família - } \\
\text { CONFTF ( } 3 \text { perguntas }\end{array}$ & Rego \& Souto (2004) & \\
\hline Estresse - ESTRES (5 perguntas) & Paschoal \& Tamayo (2004) & \\
\hline
\end{tabular}

Quadro 1. Constructos da Pesquisa (JUSTORG - CONFTF - ESTRES)

Fonte: Dados da pesquisa (2019).

Para elucidar essa relação, foi empregado a técnica de modelagem de equações estruturais (MEE) com utilização do software Amos, versão 21. Neste estudo, a modelagem por equações estruturais, não propõe à validação de constructos ou dimensões, e nem testar a confiabilidade dos indicadores, uma vez que as variáveis possuem apenas uma escala de medida (Escala Likert - 7 pontos). Portanto, a modelagem de equações estruturais apenas testa as relações de causalidade e fornece índices que verificam o ajuste do modelo à base de dados testada (Marôco, 2010). O quadro 2 apresenta os índices de ajustes absolutos, parcimônia, análise de confiabilidade e validade discriminante.

\begin{tabular}{|c|c|c|c|}
\hline \multicolumn{3}{|c|}{ ÍNDICES DE AJUSTES ABSOLUTO } \\
\hline Indicadores & Descrição & $\begin{array}{c}\text { Valores } \\
\text { Adequados }\end{array}$ & Autores \\
\hline $\begin{array}{c}\text { Quociente } \\
\text { Liberdade }(G L)\end{array}$ & $\begin{array}{c}\text { Indica as disparidades entre as matrizes } \\
\text { estimadas e as observadas, sugerindo, em } \\
\text { valor absoluto, que as diferenças entre as }\end{array}$ & $\leq 5$ & \\
\hline
\end{tabular}




\begin{tabular}{|c|c|c|c|}
\hline & $\begin{array}{l}\text { matrizes serão menores quanto menor for a } \\
\text { relação entre graus qui-quadrado }\left(x^{2}\right)(G L) \text {. } \\
\text { Porém, como o qui-quadrado é sensível ao } \\
\text { tamanho da amostra, sua análise somente faz } \\
\text { sentido quando os graus de liberdade são } \\
\text { considerados. }\end{array}$ & & \multirow{4}{*}{$\begin{array}{c}\text { Marôco } \\
\text { (2010) } \\
\text { Hair et al } \\
\text { (2014) }\end{array}$} \\
\hline$p$ - Significância & Indica a significância do modelo. & $<0,05(* * *)$ & \\
\hline $\begin{array}{l}\text { Parsimony-adjusted GFI } \\
\text { (GFI) }\end{array}$ & $\begin{array}{c}\text { Os índices de ajuste parcimonioso fornecem } \\
\text { informações sobre qual modelo é melhor } \\
\text { dentre um conjunto de modelos concorrentes, } \\
\text { considerando o ajuste relativo a sua } \\
\text { complexidade e o número de coeficientes para } \\
\text { análise. }\end{array}$ & $\begin{array}{l}\text { Sem valor } \\
\text { mínimo }\end{array}$ & \\
\hline $\begin{array}{l}\text { Root Mean Square Error } \\
\text { of Approximation } \\
\text { (RMSEA) }\end{array}$ & $\begin{array}{c}\text { Utilizado para verificar a correção da tendência } \\
\text { apresentada pelo }{ }_{x}^{2} \text { de rejeitar o modelo a } \\
\text { partir de grandes amostras ou grande número } \\
\text { de variáveis observadas, ou seja, mostra a } \\
\text { qualidade de ajuste do modelo à matriz de } \\
\text { covariância da amostra. }\end{array}$ & $<0,08$ & \\
\hline $\begin{array}{l}\text { Tucker-Lewis Coefficient } \\
\text { (TLI) }\end{array}$ & $\begin{array}{c}\text { Apresenta uma medida de parcimônia entre os } \\
\text { índices do modelo proposto e do modelo nulo. } \\
\text { Varia de zero a um. }\end{array}$ & $>0,90$ & \multirow{6}{*}{$\begin{array}{l}\text { Hair et al } \\
(2010)\end{array}$} \\
\hline $\begin{array}{l}\text { Comparative Fit Index } \\
\qquad(C F I)\end{array}$ & $\begin{array}{c}\text { Compara de forma geral o modelo estimado e } \\
\text { o modelo nulo, considerando valores mais } \\
\text { próximos de um como indicadores de } \\
\text { ajustamento satisfatório, ou seja, mostra se é e } \\
\text { em que medida a qualidade do ajustamento } \\
\text { do modelo proposto é melhor que a do } \\
\text { modelo de base. }\end{array}$ & $>0,90$ & \\
\hline Alfa de Cronbach & $\begin{array}{c}\text { O coeficiente a é uma estimativa da } \\
\text { confiabilidade de uma medida não considera } \\
\text { os erros nos indicadores. }\end{array}$ & $>0,7$ & \\
\hline Confiabilidade Composta & $\begin{array}{c}\text { É uma medida de consistência interna dos } \\
\text { itens. }\end{array}$ & $>0,7$ & \\
\hline Variância Média Extraída & $\begin{array}{l}\text { Representa uma medida de confiabilidade que } \\
\text { indica a quantidade geral de variância nos } \\
\text { indicadores explicada pelo constructo latente. }\end{array}$ & $>0,5$ & \\
\hline $\begin{array}{l}\text { Critério de Fornell e } \\
\text { Larcker (1981) }\end{array}$ & $\begin{array}{l}\text { Compara-se os percentuais de variância } \\
\text { extraída para dois constructos quaisquer com } \\
\text { o quadrado da estimativa de correlação entre } \\
\text { tais constructos. }\end{array}$ & $\begin{array}{l}\text { As } \\
\text { estimativas } \\
\text { de AVE } \\
\text { devem ser > } \\
\text { à estimativa } \\
\text { quadrática } \\
\text { da } \\
\text { correlação. }\end{array}$ & \\
\hline
\end{tabular}

Quadro 2. Índices de Ajustes

Fonte: Dados da pesquisa (2019).

Desse modo, o tópico seguinte irá tratar da apresentação dos resultados e discussões referente as dimensões analisadas.

\section{APRESENTAÇÃO DOS RESULTADOS E DISCUSSÕES}

Diante da posse dos dados coletados na prefeitura, os primeiros resultados apresentados são os socioeconômicos, conforme a Tabela 1: 


\begin{tabular}{|c|c|c|}
\hline PERFIL & RESPONDENTES & VALORES PERCENTUAIS \\
\hline $\begin{array}{cc}\text { SEXO } & \text { Feminino } \\
\text { Masculino } \\
\text { Não desejo informar }\end{array}$ & $\begin{array}{c}111 \\
104 \\
3\end{array}$ & $\begin{array}{c}51 \% \\
48 \% \\
1 \%\end{array}$ \\
\hline $\begin{array}{c}\text { IDADE } \\
\text { Até } 18 \text { anos } \\
\text { De } 19 \text { a } 28 \text { anos } \\
\text { De } 29 \text { a } 38 \text { anos } \\
\text { De } 39 \text { a } 48 \text { anos } \\
\text { Acima de } 49 \text { anos }\end{array}$ & $\begin{array}{c}24 \\
131 \\
39 \\
19 \\
5\end{array}$ & $\begin{array}{l}11 \% \\
60 \% \\
18 \% \\
9 \% \\
2 \%\end{array}$ \\
\hline $\begin{array}{l}\text { ESCOLA } \\
\text { Ensino Médio } \\
\text { Ensino Superior } \\
\text { Especialização } \\
\text { Mestrado }\end{array}$ & $\begin{array}{c}121 \\
84 \\
12 \\
1\end{array}$ & $\begin{array}{c}55 \% \\
38 \% \\
5 \% \\
2 \%\end{array}$ \\
\hline $\begin{array}{l}\text { TRABALHO } \\
\text { Até } 5 \text { anos } \\
\text { De } 6 \text { a } 10 \text { anos } \\
\text { De } 11 \text { a } 15 \text { anos } \\
\text { De } 16 \text { a } 20 \text { anos } \\
\text { De } 21 \text { a } 29 \text { anos }\end{array}$ & $\begin{array}{c}183 \\
18 \\
4 \\
12 \\
1\end{array}$ & $\begin{array}{l}84 \% \\
8 \% \\
2 \% \\
5 \% \\
1 \%\end{array}$ \\
\hline $\begin{array}{c}\text { ESTADO CIVIL } \\
\text { Solteiro } \\
\text { Casado } \\
\text { Divorciado } \\
\text { Outros } \\
\end{array}$ & $\begin{array}{c}168 \\
37 \\
10 \\
3\end{array}$ & $\begin{array}{l}77 \% \\
17 \% \\
4 \% \\
2 \% \\
\end{array}$ \\
\hline $\begin{array}{c}\text { RENDA } \\
\text { Até } 1.874,00 \\
\text { De } 1.874,00 \text { até } 3.748,00 \\
\text { De } 3.748,01 \text { até } 9.370,00 \\
\text { De } 9.370,01 \text { até } 18.740,00\end{array}$ & $\begin{array}{c}119 \\
68 \\
26 \\
5\end{array}$ & $\begin{array}{c}54 \% \\
31 \% \\
12 \% \\
3 \%\end{array}$ \\
\hline
\end{tabular}

Tabela 1. Resultados descritivos da pesquisa

Fonte: Dados da pesquisa (2019).

Quanto ao perfil dos 218 respondentes, a maioria, (51\%) são do sexo feminino, (60\%) possuem idade entre 19 a 28 anos e (55\%) possuem escolaridade em nível médio. Já quando se trata de trabalho, estado civil e renda, 183 está até 5 anos na função de servidor público, (77\%) é solteiro e (55\%) ganha até R\$1.874,00, respectivamente. Logo após saber-se do perfil dos respondentes, partiu-se para o refinamento das três dimensões, justiça organizacional, estresse e conflito trabalho - família, onde é apresentado na tabela 2:

\begin{tabular}{c|cc|ccc}
\hline \multirow{2}{*}{ DIMENSÕES } & QUESTÕES & $\begin{array}{c}\text { CARGA } \\
\text { PADRONIZADA }\end{array}$ & $\begin{array}{c}\text { ERRO } \\
\text { PADRÃO }\end{array}$ & T & SIG \\
& PUST_DIST01 & 0.766 & & & \\
\multirow{3}{*}{ DIST } & JUST_DIST02 & 0.916 & 0.083 & 14.679 & $* * *$ \\
& JUST_DIST03 & 0.883 & 0.086 & 14.099 & $* * *$ \\
& JUST_DIST04 & 0.869 & 0.087 & 13.82 & $* * *$ \\
\hline \multirow{2}{*}{ PROC } & JUST_PROC01 & 0.737 & & & \\
& JUST_PROC02 & 0.917 & 0.095 & 13.175 & $* * *$ \\
& JUST_PROC03 & 0.749 & 0.098 & 10.901 & $* * *$ \\
\hline INTER & JUST_INTER01 & 0.910 & & & \\
\end{tabular}




\begin{tabular}{c|cc|ccc} 
& JUST_INTER02 & 0.870 & 0.053 & 19.028 & $* * * *$ \\
& JUST_INTER03 & 0.768 & 0.056 & 14.811 & $* * * *$ \\
& JUST_INTER04 & 0.877 & 0.057 & 19.371 & $* * * *$ \\
\hline \multirow{5}{*}{ ESTR } & ESTRESSE01 & 0.773 & & & \\
& ESTRESSE02 & 0.835 & 0.085 & 13.138 & $* * *$ \\
& ESTRESSE03 & 0.889 & 0.083 & 14.135 & $* * * *$ \\
& ESTRESSE04 & 0.756 & 0.084 & 11.677 & $* * * *$ \\
& ESTRESSE05 & 0.792 & 0.095 & 12.329 & $* * * *$ \\
\hline \multirow{3}{*}{ CONF } & CONF_TRAB01 & 0.820 & & & \\
& CONF_TRAB02 & 0.890 & 0.076 & 14.463 & $* * *$ \\
& CONF_TRAB03 & 0.846 & 0.075 & 13.969 & $* * *$ \\
\hline
\end{tabular}

Tabela 2. Refinamento dos dados

Fonte: Dados da pesquisa (2019).

A tabela 2 apresenta, portanto, na primeira coluna as dimensões, o qual a justiça organizacional foi analisada sob a ótica da justiça distributiva, processual e interpessoal, tendo ainda a dimensão estresse e a conflito trabalho - família. Percebe-se que todas as questões de todas as dimensões obtiveram significância, com carga acima de $(0,737)$. Assim, verificou-se que a carga padronizada das variáveis observadas é igual ou maior a $(0,70)$, segundo a literatura (Hair Jr, 2005; Hair et al, 2010). Quando verificado os índices $\mathrm{X}^{2}, \mathrm{GFI}, \mathrm{CFI}$, RMSEA, os resultados são demonstrados na tabela 3:

\begin{tabular}{|c|c|}
\hline ÍNDICES & RESULTADOS \\
\hline$x^{2}$ & 461.11 \\
\hline GL & 142 \\
\hline$X^{2} / G L$ & 3.247253521 \\
\hline GFI & 0.834 \\
\hline TLI & 0.882 \\
\hline $\mathrm{CFI}$ & 0.902 \\
\hline RMSEA & 0.08 \\
\hline
\end{tabular}

Tabela 3. Verificação dos índices

Fonte: Dados da pesquisa (2019).

Na verificação dos índices (tabela 3), percebe-se que os resultados estão de acordo com Byrne (2013) e Hair et al, (2014), o qual ressalta que o GFI (índice de qualidade de ajuste varia de 0 (ajuste fraco) a 1 (ideal) e permite a comparação dos resíduos das matrizes observadas e estimadas) precisa estar acima de $(0,90)$. Os índices de comparação, TLI (medida de parcimônia entre os índices do modelo proposto e do modelo nulo, 0 qual varia de zero a um) e o CFI (compara de forma geral o modelo estimado e o modelo nulo, considerando valores mais próximos de um como indicadores de ajustamento satisfatório), um deles precisa estar acima de $(0,90)$. O índice de parcimônia, RMSEA (utilizado para verificar a correção da tendência apresentada pelo $x^{2}$ de rejeitar o modelo a partir de grandes amostras ou grande número de variáveis observadas), precisa estar entre $(0,04$ e 0,08$)$ (Hair et al, 2010).

Quando se trata da verificação dos índices de confiabilidade, Alfa de Cronbach (estimativa da confiabilidade de uma medida que não considera os erros nos indicadores) e Confiabilidade Composta (medida de consistência) interna dos itens), todos superiores a $(0,70)$, como recomendado pela literatura consultada (Marôco, 2011). Quanto a Variância Extraída - AVE (medida de confiabilidade que indica a quantidade geral de variância nos indicadores explicada pelo constructo latente) precisa ser maior que $(0,5)$, portanto, a (tabela 4) esboça estes resultados para as dimensões estudadas. 


\begin{tabular}{c|c|c|c}
\hline DIMENSÕES & $\begin{array}{c}\text { ALFA DE } \\
\text { CRONBACH }\end{array}$ & CONFIABILIDADE COMPOSTA & AVE \\
\hline Justiça Distributiva & 0.918 & 0.920 & 0.740 \\
\hline Justiça Processual & 0.831 & 0.850 & 0.650 \\
\hline Justiça Interpessoal & 0.915 & 0.920 & 0.740 \\
\hline Estresse & 0.903 & 0.910 & 0.660 \\
\hline Conflito Trabalho - Família & 0.887 & 0.890 & 0.730 \\
\hline
\end{tabular}

Tabela 4. Índices de confiabilidade

Fonte: Dados da pesquisa (2019).

A tabela 5 descreve os resultados da validade discriminante, que conforme Fornell \& Larcker (1981) compara os percentuais de variância extraída para dois constructos quaisquer com o quadrado da estimativa de correlação entre tais constructos, ou seja, testa os constructos em pares, calcula a diferença de quiquadrado comparando dois modelos: (1) constructos correlacionados; (2) modelos não correlacionados. Veja na tabela a seguir:

\begin{tabular}{c|r|r|r|r|r}
\hline & JUST DIST & JUST PROC & JUST INTER & ESTRESSE & $\begin{array}{c}\text { CONFLITO TRAB / } \\
\text { FAM }\end{array}$ \\
\hline JUST DIST & $\mathbf{0 . 8 6 0}$ & & & & \\
\hline JUST PROC &, $457^{* *}$ & $\mathbf{0 . 8 0 6}$ & & & \\
\hline JUST INTER &, $457^{* *}$ &, $790^{* *}$ & $\mathbf{0 . 8 6 0}$ & & \\
\hline ESTRESSE &,$- 237^{* *}$ &,$- 533^{* *}$ &,$- 582^{* *}$ & $\mathbf{0 . 8 1 2}$ & \\
\hline $\begin{array}{c}\text { CONFLITO TRAB } \\
\text { / FAM }\end{array}$ & -.065 &,$- 190^{* *}$ &,$- 169^{*}$ &, $263^{* *}$ & $\mathbf{0 . 8 5 4}$ \\
\hline
\end{tabular}

**. A correlação é significativa no nível 0,01 (2 extremidades).

*. A correlação é significativa no nível 0,05 (2 extremidades).

Tabela 5. Validade discriminante

Fonte: Dados da pesquisa (2019).

Conforme a literatura indica, os constructos correlacionados devem sempre ser superior que os não correlacionados (Fornell \& Larcker, 1981). Assim, após as estatísticas de validade do constructo, parte-se para os resultados do modelo estrutural da pesquisa. A tabela 5 apresenta os resultados do modelo com as relações entre as variáveis.

\begin{tabular}{c|c|c|c|c|c|c}
\hline $\begin{array}{c}\text { VARIÁVEL } \\
\text { INDEPENDENTE }\end{array}$ & $\begin{array}{c}\text { VARIÁVEL } \\
\text { DEPENDENTE }\end{array}$ & $\begin{array}{c}\text { PESO DA } \\
\text { REGRESSÃo }\end{array}$ & $\begin{array}{c}\text { ERRO } \\
\text { PADRÃO }\end{array}$ & T & SIG & $\mathbf{R}^{2}$ \\
\hline Justiça & Estresse & -0.624 & 0.233 & -5.813 & $* * *$ & 0.39 \\
\hline Estresse & Conflito & 0.285 & 0.104 & 2.839 & 0.005 & \\
\hline Justiça & Conflito & -0.027 & 0.222 & -0.27 & 0.787 & 0.09 \\
\hline
\end{tabular}

Tabela 6. Resultados do modelo estrutural Fonte: Dados da pesquisa (2019).

Conforme indica a tabela 6, a justiça organizacional está associada negativamente a dimensão do estresse (-0.624), confirmando a hipótese 1 proposta. Assim, pode-se entender, que os servidores da prefeitura estudada, sentem-se mais estressados quando as ações ou os mecanismos referentes à remuneração, ao 
processo de tomada de decisão e questões interpessoais são injustas. Desse modo, esta relação se confirma com as conclusões de Mendonça \& Mendes (2005), Prestes, Mendonça \& Ferreira (2013) e Da Rocha, Ceretta \& Andretto (2016), os quais destacam que o sentimento de injustiça no trabalho faz com que o indivíduo enfrente situações que intimidam e desestimulam.

Para Ferreira et al (2018) existe uma forte propensão à exaustão emocional quando os indivíduos possuem afetos negativos dirigidos ao trabalho, tais como, os resultados encontrados por Maffia \& Pereira (2014), ao analisar o nível de intensidade do estresse ocupacional em 617 gestores públicos. Neste caso, se confirma a ideia e os resultados encontrados por Dal Corso, De Carlo, Carluccio, Girardi \& Falco (2019), Gu, Nolan \& Rowley (2019) e Colquitt (2001), quando destacam que a justiça nos processos, na distribuição e no relacionamento interpessoal é fundamental. Neste contexto, tudo isso está ligado aos resultados dentro do ambiente de trabalho público, por exemplo, satisfação no trabalho, comprometimento socialmente favorável, além de maior compromisso com suas reponsabilidades profissionais (Lim \& Loosemore, 2017).

Na perspectiva da hipótese 2, foi evidenciado que quando os servidores percebem que as ações ou os mecanismos são injustos dentro do ambiente organizacional, isso gera o estresse, que por sua vez gera o conflito em casa pelas questões do trabalho, pois o resultado é uma relação positiva significativa (0.285), confirmando a hipótese 2 . No caso da hipótese 2 , o estresse surgiu como uma variável mediadora, e mostrou que o estresse causado por questões de injustiça é capaz de mudar o comportamento dos servidores em casa com suas famílias (Ferreira et al, 2018; Molino et al, 2015). Como a relação desta tríade foi positiva e significativa, ressalta-se que atrelada a essas mudanças se insere a deterioração da qualidade de vida dos servidores.

Os resultados encontrados na hipótese 2, são muito semelhantes ao de Sousa, Paúl \& Queirós (2020) que descobriram em um estudo com profissionais da área da saúde que existem correlações positivas entre fadiga, stress, burnout e conflito trabalho - família, o que reforça a importância de uma adequada conciliação entre o trabalho e a família. Como já evidenciado por outros estudos, o estresse repercute em uma rotina diária cada vez mais desgastante e no aumento das situações consideradas estressoras, caso nada seja feito para melhorar a percepção de justiça (Chanlat, 2005; Rossi, Perrewé \& Sauter, 2005; Zille, 2011).

$\mathrm{Na}$ análise que verifica se a justiça organizacional possui alguma influência no conflito trabalho família, percebe-se que o resultado foi negativo não significante $(-0.027)$ para essa amostra. Desse modo, a hipótese 3 não pode ser confirmada, pois neste caso não foi possível evidenciar que a justiça organizacional tem influência significativa na relação com o conflito trabalho - família, dadas atuais percepções sobre as condições de trabalho dentro da prefeitura. Essa relação evidenciou mais uma vez, que só a percepção de justiça organizacional não influencia no conflito trabalho - família, mas quando entra em cena o estresse, a situação muda totalmente.

Dessa forma, é preciso que mais estudos com um olhar para as instituições públicas sejam feitos, para assim verificar se o estresse ocupacional é o principal nesta tríade, provocando nos servidores reações negativas (Paschoal \& Tamayo, 2004). Além disso, a cultura organizacional, o estilo de liderança e as atitudes da gerência também seriam fatores importantes de investigação na busca pelo equilíbrio entre as demandas do trabalho e da família. A percepção da existência desse suporte organizacional agiria como um potencializador da noção de direitos do trabalhador, reduzindo o sacrifício da vida familiar e o stress, o que poderia produzir maior satisfação no trabalho (Monteiro \& Daniel, 2017).

A figura 3 ilustra o modelo final com os valores das regressões. 


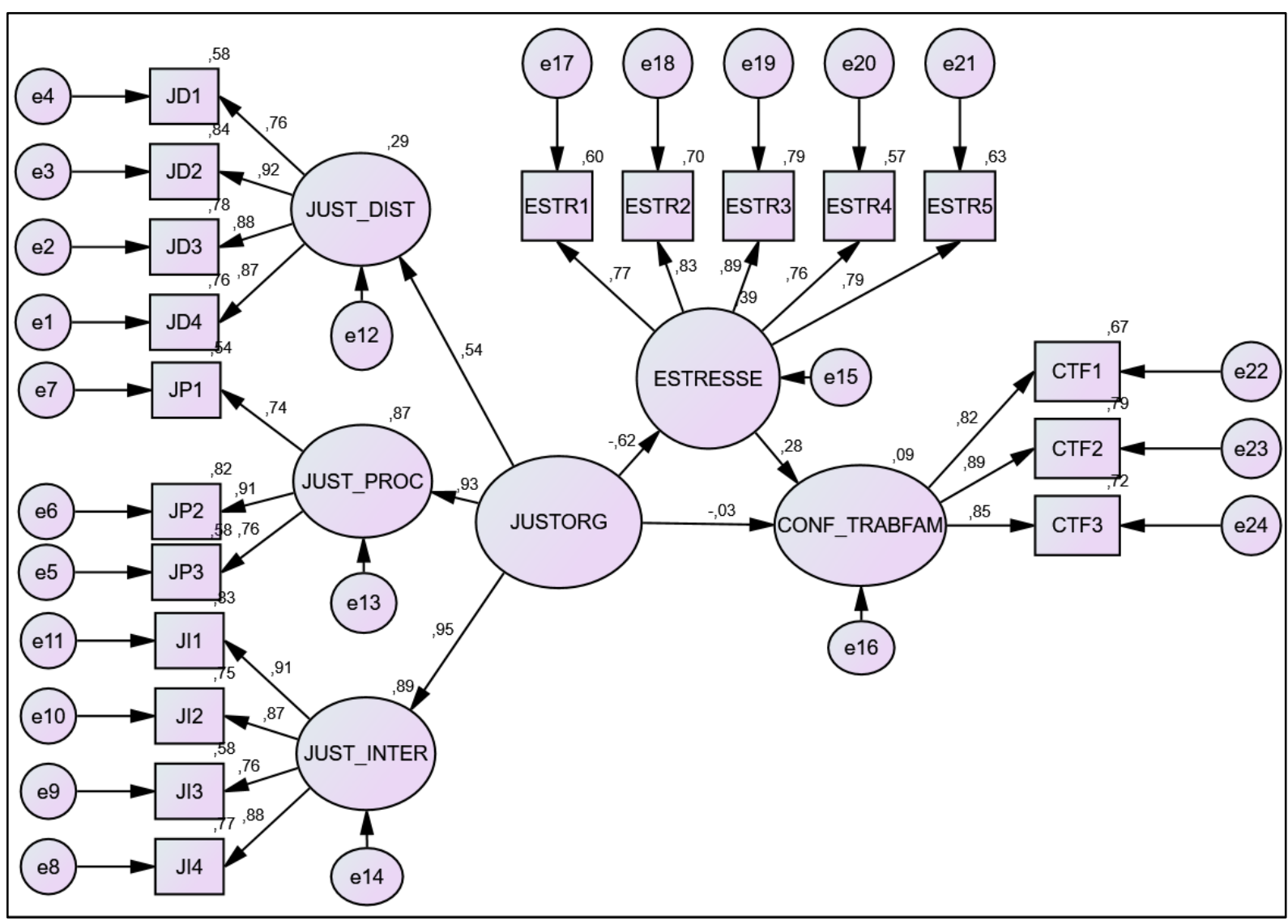

Figura 3. Modelo Estrutural Final com os Valores das Regressões

Fonte: Dados da pesquisa (2019).

Diante da apresentação e discussões dos resultados obtidos nesta pesquisa, parte-se para as considerações finais.

\section{CONSIDERAÇÕES FINAIS}

Considerando que as organizações são constituídas por pessoas, e que possuem percepções distintas quanto as formas de tratamento entre os indivíduos, em detrimento disso, sua percepção de justiça também é observada de maneira diferente (Assmar, Ferreira \& Souto, 2005). Buscando verificar a relação entre justiça organizacional, estresse e conflito trabalho - família, este estudo teve como objetivo principal, analisar a influência da justiça organizacional sobre o estresse e sobre o conflito trabalho - família, tendo como objeto de estudo, uma prefeitura da região sul do Brasil. Os principais resultados quanto ao perfil dos 218 servidores público, foi que a maior porcentagem é do sexo feminino (111), idade entre 19 e 28 anos (131) e possuem uma renda de $1.874,00$ reais $(119)$.

Quanto as associações, a justiça organizacional está associada negativamente a dimensão do estresse (-0.624), confirmando a hipótese 1 proposta. Esses resultados estão em conformidade com os achados de Ferreira et al (2018), que escrevem que existe uma forte tendência à exaustão emocional quando os indivíduos possuem afetos negativos, dirigidos ao trabalho, tais como, sentimento de tristeza, angústia e descontentamento, fazendo com que os indivíduos estejam mais vulneráveis a sentir desgaste físico e emocional.

Na segunda hipótese, foi evidenciado que quando os servidores percebem que as ações ou os mecanismos são injustos dentro do ambiente organizacional, isso gera o estresse, que por sua vez gera o conflito em casa pelas questões do trabalho, pois o resultado é uma relação positiva significativa (0.285), confirmando a hipótese 2. Nesse sentido o estudo encontrou em um setor diferente a mesma necessidade encontrada por Sousa, Paúl \& Queirós (2020), de que as organizações devem contribuir e assumir um papel essencial na identificação e prevenção destes fenómenos.

Na última hipótese (3) testada, não pode ser confirmada, pois neste estudo não se evidenciou significativamente que a justiça organizacional tem influência significativa nas relações de conflito trabalho família, dadas atuais condições de trabalho. Essa não confirmação pode ter ocorrido devido a aspectos não 
testados nesse trabalho, como os estilos de liderança e a cultura organizacional. Desse modo, Silva et al (2013) corroboram com as discussões abordadas até aqui, destacando que as atuais demandas organizacionais estão gerando cada vez mais estresse em seus trabalhadores. Neste sentido, é preciso que as empresas (públicas e privadas) adiram a mecanismos de gestão mais justos (Maissiat et al, 2015).

A contribuição que fica deste estudo, é que, quando não houver a percepção de justiça no ambiente organizacional, haverá maior probabilidade de desenvolver estresse. Estresse esse, que por sua vez vai impactar na relação trabalho - família, de seus funcionários. Como limitação deste estudo, destaca-se o fato de ter sido realizado está pesquisa em apenas uma prefeitura, não olhando para outras estruturas organizacionais, tal como universidades, empresa com ou sem fins lucrativos, para assim fazer um estudo comparativo. Como sugestão para futuras pesquisas, sugere-se que sejam identificadas associações quanto aos sexos dos respondentes, para assim comparar os resultados de percepção desta tríade entre o sexo feminino e o masculino.

Deve-se olhar para outras estruturas organizacionais, (universidades, empresas com fim lucrativo etc.) para assim fazer comparações. Além disso, investigar se estes resultados se alteram quando se olha para a idade dos respondentes, pois nesta pesquisa, as pessoas que responderam são jovens, possuem idade entre 19 a 28 anos (55\%). Um outro aspecto a ser observado, é em relação aos servidores contratados e efetivos, pois pode haver uma heterogeneidade em relação a essa tríade, comum em instituições públicas. Por fim, a tríade abordada nesta pesquisa, possui conexões com outras dimensões organizacionais, como cultura, clima organizacional, valores e desempenho, tendo espaço para ser explorado de forma integrada com justiça / estresse / conflito trabalho - família.

\section{REFERÊNCIAS}

Adams, J. S. (1965). Inequity in social exchange. In Advances in experimental social psychology (Vol. 2, pp. 267-299). Academic Press. https://doi.org/10.1016/S0065-2601(08)60108-2

Al-Zu'bi, H. A. (2010). A study of relationship between organizational justice and job satisfaction. International Journal of Business and Management, 5(12), 102. DOl: 10.5539/ijbm.v5n12p102

Assmar, E. M. L., Ferreira, M. C., \& Souto, S. D. O. (2005). Justiça organizacional: uma revisão crítica da literatura. Psicologia: reflexão e crítica, 18(3), 443-453. https://doi.org/10.1590/S010279722005000300019

Bakker, A. B., Lieke, L., Prins, J. T., \& Van der Heijden, F. M. (2011). Applying the job demands-resources model to the work-home interface: A study among medical residents and their partners. Journal of Vocational Behavior, 79(1), 170-180. https://doi.org/10.1016/j.jvb.2010.12.004

Beuren, I. M., dos Santos, V., Marques, L., \& Resendes, M. (2017). Relação entre percepção de justiça organizacional e satisfação no trabalho. Revista de Educação e Pesquisa em Contabilidade (REPeC), 11. http://dx.doi.org/10.17524/repec.v11ii0.1721

Biroli, F. (2014). Família: novos conceitos. São Paulo: Fundação Perseu Abramo.

Bohle, P., Quinlan, M., \& Mayhew, C. (2001). The health and safety effects of job insecurity. An evaluation of the evidence. https://doi.org/10.1177/103530460101200104

Burney, L. L., Henle, C. A., \& Widener, S. K. (2009). A path model examining the relations among strategic performance measurement system characteristics, organizational justice, and extra-and in-role performance. Accounting, organizations and society, 34(3-4), 305-321. https://doi.org/10.1016/j.aos.2008.11.002

Byrne, B. M. (2013). Structural equation modeling with Mplus: Basic concepts, applications, and programming. routledge.

Chanlat, J. F. (2005). Mitos e realidades sobre o estresse dos gerentes. DAVEL, E.; MELO, MCOL Gerência em ação: singularidades e dilemas do trabalho gerencial. Rio de Janeiro: Editora FGV, 29-65.

Cohen-Charash, Y., \& Spector, P. E. (2001). The role of justice in organizations: A meta-analysis. Organizational behavior and human decision processes, 86(2), 278-321. https://doi.org/10.1006/obhd.2001.2958

Colquitt, J. A. (2001). On the dimensionality of organizational justice: a construct validation of a measure. Journal of applied psychology, 86(3), 386. 
Cooper, C. L.; Sloan, S.; William, S. (1988). Occupational stress indicator - management guide. London: Windsor.

Correia, P. M. A. R., Mendes, I. D. O., \& Silva, S. R. D. S. R. M. D. (2019). A perceção de justiça na avaliação do desempenho na Administração Pública e a produtividade. Sociologia, 37, 31-50. http://dx.doi.org/10.21747/08723419/soc37a2

Cropanzano, R., Bowen, D. E., \& Gilliland, S. W. (2007). The management of organizational justice. Academy of management perspectives, 21(4), 34-48. https://doi.org/10.5465/amp.2007.27895338

Da Rocha, A. C., Ceretta, G. F., \& Andretto, V. P. (2016). Análise da percepção de justiça no trabalho: o caso de uma instituição pública. Navus-Revista de Gestão e Tecnologia, 6(3), 97-110.

da Silva Schuster, M., da Veiga Dias, V., \& Battistella, L. F. (2015). Burnout e justiça organizacional: Um estudo em servidores públicos federais. Revista de Administração IMED, 4(3), 330-342. https://doi.org/10.18256/2237-7956/raimed.v4n3p330-342

Dal Corso, L., De Carlo, A., Carluccio, F., Girardi, D., \& Falco, A. (2019). An Opportunity to Grow or a Label? Performance Appraisal Justice and Performance Appraisal Satisfaction to Increase Teachers' WellBeing. Frontiers in Psychology, 10, 2361-2361. https://doi.org/10.3389/fpsyg.2019.02361

Edwards, J. R., \& Rothbard, N. P. (2000). Mechanisms linking work and family: Clarifying the relationship between work and family constructs. Academy of management review, 25(1), 178-199. https://doi.org/10.5465/amr.2000.2791609

Elovainio, M., Kivimäki, M., \& Vahtera, J. (2002). Organizational justice: evidence of a new psychosocial predictor of health. American journal of public health, 92(1), 105-108.

Ferreira, J. M. P., Paiva, K. C. M. D., Santos, J. N., \& Dutra, M. R. S. (2018). Estresse, retaliação e percepção de injustiça nas organizações: proposição de modelo teórico integrativo. Cadernos EBAPE. BR, 16(4), $774-$ 787. https://doi.org/10.1590/1679-395167310

Fornell, C., \& Larcker, D. F. (1981). Evaluating structural equation models with unobservable variables and measurement error. Journal of marketing research, 18(1), 39-50. https://doi.org/10.1177/002224378101800104

Garcia, P. S. D., Macadar, M. A., \& Luciano, E. M. (2018). A influência da injustiça organizacional na motivação para a prática de crimes cibernéticos. JISTEM-Journal of Information Systems and Technology Management, 15. https://doi.org/10.4301/s1807-1775201815002

Ghislieri, C., Martini, M., Gatti, P., \& Colombo, L. (2011). The "bright side" of the work-family interface: A brief work-family enrichment scale in a sample of health professionals. TPM-Testing, Psychometrics, Methodology in Applied Psychology, 18(4), 211-230.

Greenberg, J. (1982). Approaching equity and avoiding inequity in groups and organizations. In Equity and justice in social behavior (pp. 389-435). Academic Press. https://doi.org/10.1016/B978-0-12-2995804.50017-3

Greenberg, J. (1993). The intellectual adolescence of organizational justice: You've come a long way, maybe. Social Justice Research, 6(1), 135-148. https://doi.org/10.1007/BF01048736

Greenberg, J. (1993). The social side of fairness: Interpersonal and informational classes of organizational justice. lustice_in_the_workplace i-Approaching_fairness_in human resource management. Hillsdale, $\mathrm{NJ}$ : Lawrence Erlbaum Associates.

Greenhaus, J. H., \& Beutell, N. J. (1985). Sources of conflict between work and family roles. Academy of management review, 10(1), 76-88. https://doi.org/10.5465/amr.1985.4277352

Gu, F., Nolan, J., \& Rowley, C. (2020). Organizational justice in Chinese banks: understanding the variable influence of guanxi on perceptions of fairness in performance appraisal. Asia Pacific Business Review, 26(2), 169-189. https://doi.org/10.1080/13602381.2019.1700622

Hair Jr, J. F., Gabriel, M. L. D. D. S., \& Patel, V. K. (2014). Modelagem de Equações Estruturais Baseada em Covariância (CB-SEM) com o AMOS: Orientações sobre a sua aplicação como uma Ferramenta de Pesquisa de Marketing. Revista Brasileira de Marketing, 13(2), 44-55.

HAIR, J. (2005). Fundamentos de métodos de pesquisa em administração, 2005. 
Hair, J. F., Black, W. C., Babin, B. J., Anderson, R. E., \& Tatham, R. L. (2010). Análise multivariada de dados. Bookman Editora.

Hartman, S. J., Yrle, A. C., \& Galle, W. P. (1999). Procedural and distributive justice: Examining equity in a university setting. Journal of Business Ethics, 20(4), 337-352. https://doi.org/10.1023/A:1006102216883

Höffe, O. (2003). O que é justiça? (Vol. 155). Edipucrs.

Homans, G. C. (1961). Social Behavior. Its Elementary Forms Harcourt. Brace \& World, New York.

Judge, T. A., \& Colquitt, J. A. (2004). Organizational justice and stress: the mediating role of work-family conflict. Journal of applied psychology, 89(3), 395.

Kline, R. B (2005). Principles and practice of structural equation modeling. New York: The Guilford Press.

Lim, B. T., \& Loosemore, M. (2017). The effect of inter-organizational justice perceptions on organizational citizenship behaviors in construction projects. International Journal of Project Management, 35(2), 95106. https://doi.org/10.1016/j.ijproman.2016.10.016

Lind, E. A., \& Tyler, T. R. (1988). The social psychology of procedural justice. New York: PlenumLocke, EA, \& Schweiger, DM (1979) Participation in decision-making: One more look. Research in Organizational Behavior, 32(1), 265-339.

Maffia, L. N., \& Pereira, L. Z. (2014). Estresse no trabalho: estudo com gestores públicos do estado de minas gerais. REAd. Revista Eletrônica de Administração (Porto Alegre), 20(3), 658-680.

Maissiat, G. D. S., Lautert, L., Pai, D. D., \& Tavares, J. P. (2015). Contexto de trabalho, prazer e sofrimento na atenção básica em saúde. Revista Gaúcha de Enfermagem, 36(2), 42-49. https://doi.org/10.1590/19831447.2015.02.51128

Marôco, J. (2010). Análise de Equações Estruturais: Fundamentos teóricos, software \& Aplicações. ReportNumber, Lda.

Marôco, J. (2011). Análise estatística com o SPSS Statistics [Statistical analysis with SPSS Statistics]. Pero Pinheiro, Portugal: ReportNumber.

Mendonça, H., \& Mendes, A. M. (2005). Experiências de injustiça, sofrimento e retaliação no contexto de uma organização pública do estado de Goiás. Psicologia em estudo, 10(3), 489-498. https://doi.org/10.1590/S1413-73722005000300017

Merton, R. K. (1957). The role-set: Problems in sociological theory. The British Journal of Sociology, 8(2), 106120.

Molino, M., Bakker, A. B., \& Ghislieri, C. (2016). The role of workaholism in the job demands-resources model. Anxiety, Stress, \& Coping, 29(4), 400-414. https://doi.org/10.1080/10615806.2015.1070833

Monteiro, A. C. F., \& Mourão, L. (2016). Resiliência e justiça organizacional como antecedentes da percepção de desenvolvimento profissional. Psicologia: Teoria e Pesquisa, 32(1). https://doi.org/10.1590/010237722016012402111121

Monteiro, R., \& Daniel, F. (2017). The Influence of Organizational Reconciliation Policies and Culture on Workers Stress Perceptions. Brazilian Administration Review 14(3), 1-13.

Nojani, M. I., Arjmandnia, A. A., Afrooz, G. A., \& Rajabi, M. (2012). The study on relationship between organizational justice and job satisfaction in teachers working in general, special and gifted education systems. Procedia-Social and Behavioral Sciences, 46, 2900-2905. https://doi.org/10.1016/j.sbspro.2012.05.586

Oliveira, A. B. S. et al (2003). Métodos e Técnicas de Pesquisa em Contabilidade. São Paulo: Saraiva.

Omar, A. (2006). Justicia organizacional, individualismo-colectivismo y estrés laboral. Psicología y salud, 16(2), 207-217. 
Paschoal, T., \& Tamayo, Á. (2004). Validação da escala de estresse no trabalho. Estudos de Psicologia (Natal), 9(1), 45-52. https://doi.org/10.1590/\$1413-294X2004000100006

Prestes, L. I. N., Mendonça, H., \& Ferreira, M. C. (2013). Por que a Percepção de Justiça Organizacional torna os Trabalhadores mais Comprometidos?. Revista Fragmentos de Cultura-Revista Interdisciplinar de Ciências Humanas, 23(3), 319-333. http://dx.doi.org/10.18224/frag.v23i3.2953

Rawls, J. (2008). Uma teoria da justiça. Tradução de Jussara Simões.

Rego, A. (2000). Comportamentos de cidadania organizacional: uma abordagem empírica a alguns dos seus antecedentes e consequências. Comportamento Organizacional e Gestão. v. 6, n. 12, p. 161-197.

Rego, A. (2002). Comprometimento afectivo dos membros organizacionais: o papel das percepções de justiça. Revista de Administração Contemporânea, 6(2), 209-241. https://doi.org/10.1590/S141565552002000200012

Rego, A., \& Souto, S. (2004). A percepção de justiça como antecedente do comprometimento organizacional: um estudo luso-brasileiro. Revista de administração contemporânea, 8(1), 151-177. https://doi.org/10.1590/S1415-65552004000100008

Rossi A. M.; Perrewé P. L.; Sauter S. L. (Org.) (2005). Stress e qualidade de vida no trabalho. São Paulo: Atlas.

Sadir, M. A., \& Lipp, M. E. N. (2009). As fontes de stress no trabalho. Revista de Psicologia da IMED, 1(1), 114 126. https://doi.org/10.18256/2175-5027/psico-imed.v1n1p114-126

Silva, E. P., Minette, L. J., Souza, A. P., Marçal, M. A., \& Sanches, A. L. P. (2013). Fatores organizacionais e psicossociais associados ao risco de LER/DORT em operadores de máquinas de colheita florestal. Revista Árvore, 37(5), 889-895. https://doi.org/10.1590/S0100-67622013000500011

Sousa, L. M., Paúl, C., \& Queirós, C. (2020). Fadiga por compaixão, burnout e conflito trabalho-família em profissionais de saúde.

Tepper, B. J. (2001). Health consequences of organizational injustice: Tests of main and interactive effects. Organizational behavior and human decision processes, 86(2), 197-215. https://doi.org/10.1006/obhd.2001.2951

Tyler, T. R., Boeckmann, R. J., Smith, H, Huo, A.Y. J. (1997). Social justice in a diverse society. Colorado: Westview Press.

Zille, L. P. (2011). O estresse e os impactos no trabalho na função gerencial: buscando as interfaces da realidade brasileira e portuguesa. In: BARBOSA, A. C.; SILVA, J. R. (Org.). Economia, gestão e saúde. Lisboa: Colibri, p. 137-169. 